\title{
Postoperative analgesic efficacy of bupivacaine with hyalourindase in TAP block for lower abdominal surgeries
}

\section{Salah Ahmed Mohamed*Fawzy Abbass Badawy**Hitham Mohamed}

\author{
Aly***Ghada Mohamed Refat***** \\ *Professor of Anesthesia \&ICU **Lecturer of anesthesia \&ICU ***Lecturer of \\ anesthesia \& ICU ****M.B.B.Ch of Anesthesia \&ICU
}

\begin{abstract}
Aim : To evaluate the effect of addding hyalourindase to bupivacaine in TAP block for lower abdominal surgeries as postoperative analgesia.

Methods : fifty patients aged between 20-60 years who were scheduled for lower abdominal surgeries included in the study and divided into two groups, after approval of ethical committee. This study conducted in Sohag university hospitals from September 2016 to March 2017.

Patients were divided into two equal groups and all received spinal anesthesia :(Group A) 25 patients received $20 \mathrm{ml}$ bupivacaine injected in the plane between internal oplique and transversus abdominis musceles.(Group B) 25 patients received $20 \mathrm{ml}$ bupivacaine added to 750 unit hyalourindase. Patients were monitored for heart rate NIBP and oxygen saturation .In post -anesthesia care unit patients were asked to assess their level of pain based on visual analog scale (VAS), complications also were observed .
\end{abstract}

\section{Results :}

Our study showes that addition of hyalourindase to bupivacaine for TAP block resulted in significant reduction in VAS pain score over the post operative $24 \mathrm{hrs}$ and reduction of post operative morphine requirments .

\section{Introduction}

Pain is one of the main postoperative patient complaints which may prolong the period of hospital stay and increase incidence of complications.Modern multimodal analgesia concepts have been demonstrated to provide postoperative analgesia as equally as intravenous opioid in lower abdominal surgeries, TAP block is relatively new regional anesthesia technique. ${ }^{1}$ The transversus abdominis plane (TAP) block is a peripheral nerve block that provide analgesia to the anterior abdominal wall using surface anatomic land marks or ultrasound guidance, local anesthetic injected into the neurofascial plane between the internal oblique and transversus abdominis muscle. ${ }^{2-4}$ The main goal of this technique is to reduce the amount of opioid medication used in postoperative analgesia together with its adverse effects as sedation, nausea, vomiting and urinary retention ${ }^{5}$ Several adjuvants have been added to local anesthetics to improve quality of analgesia as (adrenaline, dexamethasone and hyalourindase) ${ }^{6}$ Hyaluronidase is an enzyme that 
hydrolyses hyaluronic acid in the tissue

It has been shown to aid the spread of local anaethestic through tissue ${ }^{7}$. It is postulated that it achieves this by a reversible depolymerizing effet on hyaluronic acid. ${ }^{8}$ Hyaluronidase is commonly used as a local anaethestic adjunct in ophthalmicanaesthesia and its use was first described in $1949^{9}$. It is thought that patients may experience better analgesia through the postoperative period $10 \quad$ We hypothesize that adding hyalourindase to bupivacaine in TAP block may improve the analgesic efficacy of this technique.

\section{Patients and methods}

After approval of ethical committee, this prospective randomized double blind study will be conducted in Sohag university hospital, fifty patients scheduled for lower abdominal surgery will be included in the study and divided into two groups (group A no.25, group B no 25). Written informed consent will be obtained from all patients.After establishment of intravenous access and placement of standerd monitoring (ECG, noninvasive blood pressure, pulse oximetry and capnography.All patients will receive spinal anesthesia using Quincki needle 22 gauge, local anesthetic bupivacaine $0.5 \% 3 \mathrm{ml}$.All patients will be placed in supine position .After skin preparation, linear ultrasound probe (Sonoscape A5, SonoScape Co., Ltd.,China) is placed in transverse plane between the lower costal margin and the iliac crest in the mid axillary line, 18 gauge Touhyneedle (Perican B.Braun Melsungen AG, GERMANY) is advanced using in-plane technique with an antro- medial to postro- lateral direction, the needle is advanced until the tip is placed in the plane between the internal oblique and transversus abdominis muscels, the local anesthetic is deposited and seen as ahypoechoic shadow pushing the 2 layers apart ${ }^{11} \operatorname{group}(\mathrm{A}) \quad 20 \mathrm{ml}$ Bupivacaine Hydrochloride $0.25 \%$ group ( B) $20 \mathrm{ml}$ Bupivacaine Hydrochloride $0.25 \%$ added to it 750 $\mathrm{U}$ hyalourindase . After surgery all patients will be transferred to post anesthesia care unit to be assessed at 2,4,6,8 12, 24 hours post operatively for visual analog scale at rest and physical activity (ranging from (0) no pain to (10) maximal pain), time to first analgesia, consumption of rescue analgesia, vital signs (pulse, B.P, and oxygen saturation) and patient satisfaction .All patients will receive paracetamole $1 \mathrm{~g}$ one hour after surgery and will be allowed to receive ketolarac $30 \mathrm{mg}$ as a rescue analgesia if visual analogue scale is more than 3 .

\section{Statistical analysis}

Data will be analyzed using statistical program for social sciences IBM-SPSS (version19, Chicago, USA) program.

Parametric data (continuous or discrereet) will be analyzed using oneway ANOVA.

Evaluation of non-parametric data (nominal or ordinal) will be analyzed using Chi-Square test.

\section{Results}

Our study showes that addition of hyalourindase to bupivacaine for TAP block resulted in significant reduction in VAS pain score over the post operative $24 \mathrm{hrs}$ and reduction of post operative morphine requirments .. 


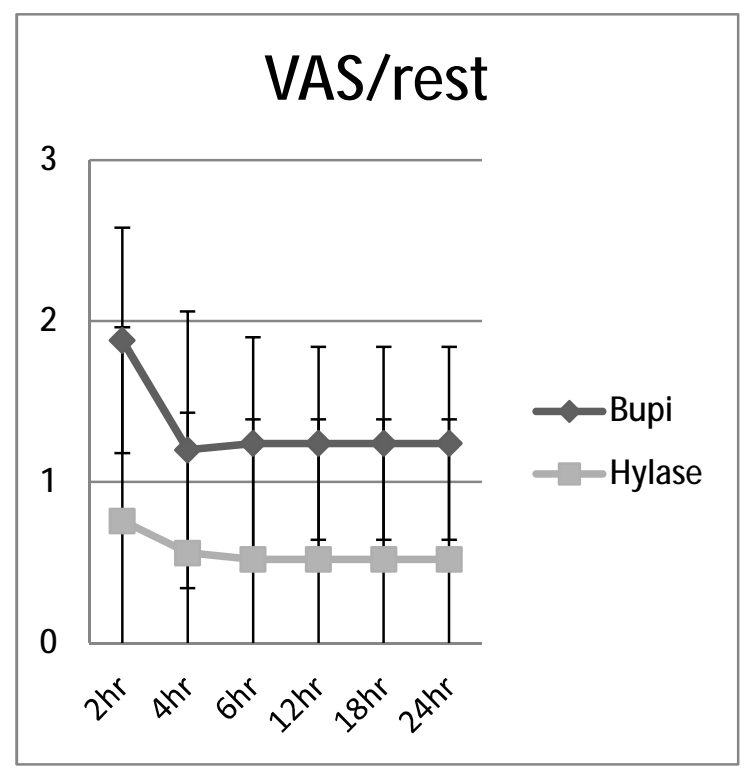

VAS score at rest decreased significantly compared to VAS $2 \mathrm{hr}$ in both groups (Pvalue $=<0.001$ ). There was significant decrease in VAS score at rest in hylase group $(\mathrm{P}$-value $=0.035)$.

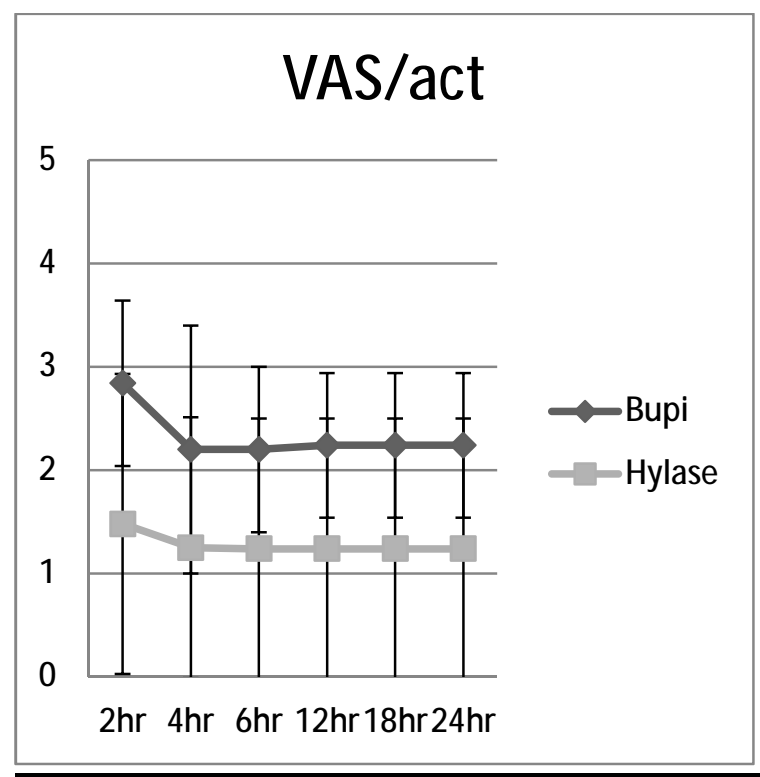

VAS active:

VAS score at activity decreased significantly compared to VAS $2 \mathrm{hr}$ in both groups (Pvalue $=<0.001)$. There was a decrease in VAS score at activity in hylase group but not statistically significant $(\mathrm{P}$-value $=0.21)$.

\section{Discussion}

Our study shows efficacy of addition of hyalourindase to bupivacaine in Transversus abdominis plane(TAP) block for post operative analgesia in lower abdominal surgeries. The transversus abdominis plane (TAP) 
block is a regional anesthesia technique that provides analgesia to the parietal peritoneum as well as the skin and muscles of the anterior abdominal wall. First described just a decade ago ( Rafi,2001), it has undergone several modifications, which have highlighted its potential utility for an increasing array of surgical procedures. Despite a relatively low risk of complications and a high success rate using modern techniques, TAP blocks remain overwhelmingly underutilized (Charlton et al,2010). The block provides an opioid-sparing effect, but the effects on opioid-related side effects, postoperative nausea and vomiting, and bowel function are not fully consistent. The effects on early postoperative pain and reduced opioid consumption 24-48 hours after surgery are seemingly similar to single shot spinal anaesthetic with intrathecal morphine. Many adjuvants have been added to local anesthtics to increase the analgesic efficacy and duration, post operative such as dexamethasone,adrenaline,dexametomiden ,clonidin and hyalourindase Tandoc et al 2011. Hyalourindase has been used successfully as a spreading factor for local anesthetics in ophthalamic surgeries for more than 60 years Hobby et al 1941 . Studies have shown increased absorption and dispersion of drugs used in combination with hyaluronidase without an increase in incidence or severity of side effects of the coadministered drugs compared with when the drugs are administered alone. All injectable formulations generally have identical indications, activities and dosing,up to our knowledge hyalourindase hasn,t been used with TAP block before.One of the earliest prospective randomized controlled trials about the efficacy of TAP block published in late 2000's. McDonnell et al. declared that TAP block was found "highly" effective in postoperative wound pain relief. According to the study results, in TAP block group VAS scores has been reduced at all post-operative time points and at the $24^{\text {th }}$ hour after surgery mean VAS scores were lower. As different from our study, the authors also evaluated the patients' post-operative sedation scores which were reduced at $4^{\text {th }}$ and $6^{\text {th }}$ hour after surgery. However, our results confirm the effectiveness of TAP block especially at the $24^{\text {th }}$-postoperative-hour regarding the VAS scores. In a similar study, Niraj et al 2009 reported that TAP block has reduced morphine consumption at the $24^{\text {th }}$ post-operative hour and found significantly a lower pain scores at rest and on coughing in the TAP block group comparing with standard pain care group.

Regarding the postoperative duration of analgesic effect of TAP block, Many issues postulated that it provides effective early (0-12 h) up to 24 or even 36 hours postoperative analgesia after a lower abdominal surgery. However, whether either technique produces prolonged analgesia lasting beyond $12 \mathrm{~h}$ remains controversial. Mc Donnell et al 2008 demonstrated that the TAP blocks reduced overall postoperative morphine requirements by more than $70 \%$ in the first 48 postoperative hours. The finding that the TAP block reduced morphine requirement for each 12 hourly interval up to $36 \mathrm{~h}$ is of importance, in that it demonstrates that a single-shot TAP technique can produce effective analgesia for up to 36 hours The reasons for the prolonged duration of analgesic effect after TAP blockade may relate to the fact that the TAP is relatively poorly vascularized, and therefore drug clearance may be slowed. 


\section{CONCLUSION}

TAP block is a new technique that improves analgesia and may be an effective tool of multimodal postoperative analgesia after abdominal surgery. Lots of clinical trials have demonstrated its superiority over standard protocols regarding postoperative pain control. TAP block reduces significantly the consumption of opioid in the first 24-h and increases patient's satisfaction after abdominal surgeries.

\section{References}

1- Walter CJ, Maxwell-Armstrong C, Pinkney TD, Conaghan PJ, Bedforth N,Gornall CB, Acheson AG. A randomised controlled trial of the efficacy of ultrasound-guided transversus abdominis plane (TAP) block in laparoscopic colorectal surgery. Surg Endosc 2013, 27:23662372.

2-AN Rafi, Abdominal Field Block: a New Approach Via The Lumbar Triangle; Anaesthesia; 2001; 56:10246.

3- JG McDonnell, BD O'Donnell, D Tuite, $\mathrm{T}$ Farrell, $\mathrm{C}$ Power, The Regional Abdominal Field Infiltration Technique Computerised Tomographic and Anatomical Identification of a Novel Approach to the Transversus Abdominis Neuro-Vascular Fascial Plane; Anaesthesiology; 2004; 101: A899.
4- P Hebbard, Y Fujiwara, Y Shibata,

C Royse. Ultrasound Guided Transversus Abdominis Plane Block. Anaesthesia \& Intensive Care; 2007; 35(4): 616-7.

5-Marret E, Kurdi O, Zufferey P, Bonnet $F$ : Effects of nonsteroidalantiinflammatory drugs on patient-controlled analgesia morphine sideeffects: meta-analysis of randomized controlled trials. Anesthesiology 2005,102:1249-1260.

6- Tandoc MN,Fan L,Kolesnikov S ,Kruglov A ,Nader ND .Adjuvant dexamethasone with bupivacaine prolonges the duration of block :prospective randomized trial. J Anesth .2011;25(5):704-709 .

7- Stern R, Jedrzejas MJ. Thehyaluronidases: their genomics, structures, andmechanisms of action. Chem Rev 2006;106:818-39.

8- Roberts JE, MacLeod BA, Hollands RH. Improved peribulbar anaesthesia withalkalinization and hyaluronidase. Can Anaesth 1993;40:835-8.

9-Atkinson WS. Use of hyaluronidase with local anaesthesia in ophthalmology;preliminary report. Arch Opthalmol 1949;42:628-33.

10- Carney J, Finnerty O, Rauf J, et al. Studies on the spread of local anaesthetic

solution in transversus abdominis plane blocks. Anaesthesia 2011;66:1023-30.

11- AN Rafi, Abdominal Field Block: a New Approach Via The Lumbar Triangle; Anaesthesia; 2001;56:1024- 